\title{
Características clínicas y terapeúticas de los pacientes ingresados en un Servicio de Medicina Interna por insuficiencia cardiaca
}

\author{
I. FERNÁNDEZ GALANTE, E. GONZÁLEZ SARMIENTO
}

Servicio de Medicina Interna. Hospital Clínico Universitario. Valladolid

\author{
CLINICAL AND THERAPEUTIC CHARACTERISTICS IN PATIENTS \\ WITH HEART FAILURE CARED IN AN INTERNAL MEDICINE \\ DEPARTMENT
}

\section{RESUMEN}

Introducción: La insuficiencia cardiaca (IC) es un síndrome muy frecuente en la práctica clínica y de gran importancia socio sanitaria. En España constituye, en pacientes mayores de 65 años, la primera causa de ingreso hospitalario en los Servicios de Medicina Interna (MI).

Objetivo: determinar el perfil de los pacientes ingresados en un servicio de MI por IC.

Material y métodos: Se trata de un estudio retrospectivo descriptivo. Se analizaron las siguientes variables: sexo, edad, motivo del ingreso, etiología, factores de riesgo asociados, cumplimiento de los criterios diagnósticos de Framingham, tipo de IC, tratamiento al alta, estancia media, número de reingreso y el tipo de disfunción ventricular (ecocardiografía). Método estadístico, el test Chi-cuadrado de Pearson o el test exacto de Fisher. Aquellos valores de $\mathrm{p}<0,05$ han sido considerados estadísticamente significativos.

Resultados: Se analizaron un total de 104 pacientes. El porcentaje con IC, fue del $13,76 \%$. El 52,88\% fueron hombres, con una edad media de $82,92 \pm 7,24$ años para las mujeres y $72,16 \pm 17,78$ años para los hombres. Motivo principal de ingreso: disnea $(74 \%)$. Factores de riesgo asociados: la HTA como más prevalente $(70,13 \%)$. En cuanto a la etiología el primer lugar lo ocupa la HTA con el $68,57 \%$. Los tipos de IC más frecuente fueron la global $(33,65 \%)$ y la izquierda $(33,65 \%)$. El $5,17 \%$ de las mujeres y el 20,69\% de los hombres presentaron disfunción sistólica (p 0,023). Presentaban disfunción diastólica el 32,76\% de las mujeres y el $41,38 \%$ de los hombres. Al alta la mayoría de los pacientes recibieron como tratamiento de fondo diuréticos y digitálicos. La estancia media fue de 15,49 días y los reingresos alcanzaron el $26,67 \%$.

Conclusión: El diagnóstico y el tratamiento de la IC en el servicio de MI es aceptable. Sería importante potenciar el uso de betabloqueantes e inhibidores de la aldosterona.

PALABRAS CLAVES: Insuficiencia cardiaca. Medicina Interna.

\section{ABSTRACT}

Introduction: The Heart Failure $(H F)$ is a very common clinic syndrome in the clinical practise, and a great sanitary social problem. In Spain, it is the main cause of hospitalization in Internal Medicine Services in patients over 65 years old, due to its high comorbidity, in many cases.

Objective: To determine the profile of admitted patients with HF to an Internal Medicine Service.

Material and methods: It is a retrospective and descriptive study. The following variables were analysed: sex, age, cause of admission, aetiology, risk factors, Framingham diagnostic criteria, type of HF, treatmenton-released, average stay, number of readmissions and the type of ventricular dysfunction (echocardiography). Chi-square test of Pearson or Fisher test were used as statistical methods. $P<0.05$ was considered to be statistically relevant.

Results: 104 patients were analysed. The percentage of $H F$ was $13.76 \% .52 .88 \%$ were men with an average age of $72.16 \pm 17.78$ year. Average age for women was $82.92 \pm 7.24$ year. The main cause of admission was dysnea (74\%). Hypertension was the principal risk factor $(70.13 \%)$. When it comes to aetiology, HTA (68.57\%) was the main one. Global and left HF (33.65\% either) were the most common type of HF. $5.17 \%$ of women and $20.69 \%$ of men suffered from systolic dysfunction $(p<0.023) .32 .76 \%$ of women and $41.38 \%$ of men had diastolic dysfunction. The base treatment-on-release for the majority of patients consisted of diuretics and digoxin. Patients stayed 15.49 days on average, and the number of readmitted ones came up to $26.67 \%$.

Conclusions: The diagnosis and treatment of HF in an Internal Medicine Service is acceptable. It will be very important to promote the use of beta blockers and angiotensina-converting enzyme inhibitors.

KEY WORDS: Heart Failure. Internal Medicine.

Fernández Galante I, González Sarmiento E. Características clínicas y terapéuticas de los pacientes ingresados en un Servicio de Medicina Interna por insuficiencia cardiaca. An Med Interna (Madrid) 2006; 23: 459-464.

\section{INTRODUCCIÓN}

La insuficiencia cardiaca (IC) es un síndrome muy frecuente en la práctica clínica, de gran importancia socio sanitario por su elevada prevalencia y mortalidad.
En Europa la padece el 1\% de la población (1). La prevalencia se duplica con cada década de edad y se sitúa alrededor del $10 \%$ en sujetos mayores de 70 años (2-4). No obstante, los estudios de incidencia y prevalencia presentan una amplia variabilidad por la ausencia de criterios diagnósticos unáni-

Trabajo aceptado: 9 de mayo de 2006

Correspondencia: Inmaculada Fernández Galante. Servicio de Medicina Interna (10ª planta este). Hospital Clínico Universitario. C/ Ramón y Cajal, 3. 47005 Valladolid. e-mail: inmajg@gmail.com 
memente aceptados para la identificación de esta entidad clínica.

En España, la IC constituye, en pacientes mayores de 65 años, la primera causa de ingreso hospitalario en los Servicios de Medicina Interna (4,5) debido, en muchos casos, a la alta comorbilidad asociada (diabetes, enfermedad pulmonar obstructiva crónica, hipertensión arterial, etc), lo que conlleva secundariamente a un aumento en el gasto sanitario (6).

La IC tiene un gran impacto demográfico por su elevada mortalidad como demuestra el estudio Framingham (7). Según este estudio, el $75 \%$ de los hombres y el $62 \%$ de las mujeres mueren a los 5 años de su diagnóstico. En España es la tercera causa de mortalidad por enfermedad cardiovascular, ocasionando el $25 \%$ de los fallecimientos por esta causa (2).

Hasta hace poco, su diagnóstico se basaba en criterios clínicos inespecíficos, sin tener en cuenta la disfunción ventricular sistólica o diastólica, medida mediante ecocardiograma. Esto provocaba que un número importante de pacientes no fuesen diagnosticados de IC, y otros sujetos con otras patologías, sí lo fuesen. Esto ha hecho difícil su cálculo epidemiológico (8).

Las estrategias terapéuticas para la IC crónica se basan en la depleción de líquidos e inhibición de la activación de los sistemas neurohumorales, en especial el sistema renina-angiotensina-aldosterona (SRAA) y el sistema nervioso simpático. Así, se recomienda la combinación de diuréticos, inhibidores de la enzima conversora de angiotensina (IECAs) y beta bloqueantes, con o sin digoxina, como base del tratamiento.

El objetivo de este trabajo ha sido determinar el perfil de los pacientes ingresados en un servicio de Medicina Interna por IC, su prevalencia, la etiología, los factores de riesgo, el cumplimiento de los criterios de Framingham, el tipo de disfunción ventricular, el número de reingresos, la estancia media de ingreso hospitalario y por último evaluar la actuación terapéutica en estos pacientes.

\section{MATERIAL Y MÉTODOS}

Se trata de un estudio retrospectivo descriptivo, que analiza los pacientes que con el diagnóstico de IC son atendidos en un servicio de Medicina Interna del Hospital Clínico Universitario de Valladolid (HCUV), en régimen de hospitalización.

El HCUV es de categoría intermedia (grupo 3 de la clasificación de hospitales del INSALUD) que tiene entre 6 y 8 especialidades complejas con trasplante. Cuenta con 777 camas funcionantes y atiende al área II de la provincia de Valladolid (zona este) con una población total asignada de 211.953 habitantes, de edad intermedia con tendencia al envejecimiento, según la pirámide poblacional del momento del estudio (año 2004).

\section{RECOGIDA DE DATOS}

Para el estudio contamos con los datos recogidos de los informes médicos al alta de cada uno de los pacientes de un servicio de Medicina Interna del HCUV. Se incluyeron todos aquellos en cuyo informe figuraba el diagnóstico de IC. Dicho diagnóstico se basó en los criterios de Framinghan (Tabla I) (9) considerando IC el presentar al menos 2 criterios mayores o bien 1 criterio mayor y 2 menores.
TABLA I

PRINCIPALES FACTORES DE RIESGO EN RELACIÓN CON EL SEXO

\begin{tabular}{lccc}
\hline Factor de riesgo & Hombre & Mujer & P valor \\
\hline HTA & $60 \%$ & $79,59 \%$ & 0,031 \\
Tabaco & $47,27 \%$ & $2,04 \%$ & $<0,001$ \\
EPOC & $29,09 \%$ & $2,04 \%$ & $<0,001$ \\
Hiperuricemia & $20 \%$ & $0 \%$ & $<0,001$ \\
Diabetes mellitus & $36,36 \%$ & $32,65 \%$ & 0,691 \\
Dislipemia & $23,63 \%$ & $16,32 \%$ & 0,354 \\
\hline
\end{tabular}

Tras la revisión de los informes de alta se obtuvieron 104 pacientes con el diagnóstico de IC.

Se analizaron las siguientes variables: el sexo, la edad, el motivo del ingreso (obtenido según el diagnóstico principal indicado en el informe de alta), la etiología, los factores de riesgo asociados, el cumplimiento de los criterios diagnósticos de Framingham, el tipo de insuficiencia cardiaca (según la clínica), el tratamiento al alta, la estancia media y el número de reingresos de los pacientes. El tipo de disfunción ventricular se valoró mediante ecocardiografía, considerándose como función sistólica conservada la fracción de eyección (FE) $\geq$ $50 \%$ y deprimida si la FE era $<50 \%$. Así mismo, se tipificó la disfunción diastólica mediante la valoración de hipertrofia ventricular, con fracción de eyección conservada, la dilatación auricular izquierda y la alteración en la relajación ventricular.

\section{ANÁLISIS ESTADÍSTICO}

Se ha realizado con el programa EPIDAT versión 3.1 (programa para análisis epidemiológico de datos tabulados). Se ha utilizado el test Chi-cuadrado de Pearson o el test exacto de Fisher en el caso de que el número de celdas con valores esperados menores de 5 fuera mayor de un 20\%. Aquellos valores de $\mathrm{p}<0$, 05 han sido considerados estadísticamente significativos.

\section{RESULTADOS}

Se analizaron un total de 104 pacientes ingresados en un Servicio de Medicina Interna del HCUV por IC, según los datos aportados por los informes clínicos al alta.

El porcentaje de pacientes con IC, sobre un total de 756 ingresados en este servicio durante el año 2004, fue del $13,76 \%$ (104 pacientes) y de ellos, el 52,88\% (55) fueron hombres, con una edad media de 82,92 \pm 7,24 años para las mujeres y 72,16 $\pm 17,784$ años para los hombres.

El motivo principal de ingreso fue la disnea (74\% de los pacientes), seguido de la infección respiratoria $(10,58 \%)$, edemas $(4,8 \%)$, cuadro sincopal $(2,8 \%)$ y el resto $(7,82 \%)$ lo fue por otras causas.

Como factores de riesgo asociados a la IC cabe destacar la HTA como más prevalerte, con un 70,13\%, seguido de la diabetes mellitus $(35,06 \%)$ y se encontraron diferencias significativas entre sexos con la HTA (p 0,032), la hiperuricemia, el tabaco y la EPOC ( $\mathrm{p}<0,001)$ como se muestra en la tabla II.

En cuanto a la etiología el primer lugar lo ocupa la HTA con el $68,57 \%$ de los casos, seguido de la fibrilación auricular con un $57,69 \%$ y las valvulopatías con un $44 \%$ (Fig. 1). 


\section{TABLA II}

CRITERIOS DIAGNÓSTICOS MAYORES Y MENORES DE FRAMINGHAM

\begin{tabular}{ll}
\hline Criterios mayores & Criterios menores \\
\hline Crepitantes 76,19\% & Edemas 60,95\% \\
Cardiomegalia 56,19\% & Derrame pleural 41,90\% \\
Ortopnea 39,05\% & Hepatomegalia 28,57\% \\
Ingurgitación yugular 30,48\% & Taquicardia $\geq 120$ lpm 8,5\% \\
EAP 2,86\% & \\
\hline
\end{tabular}

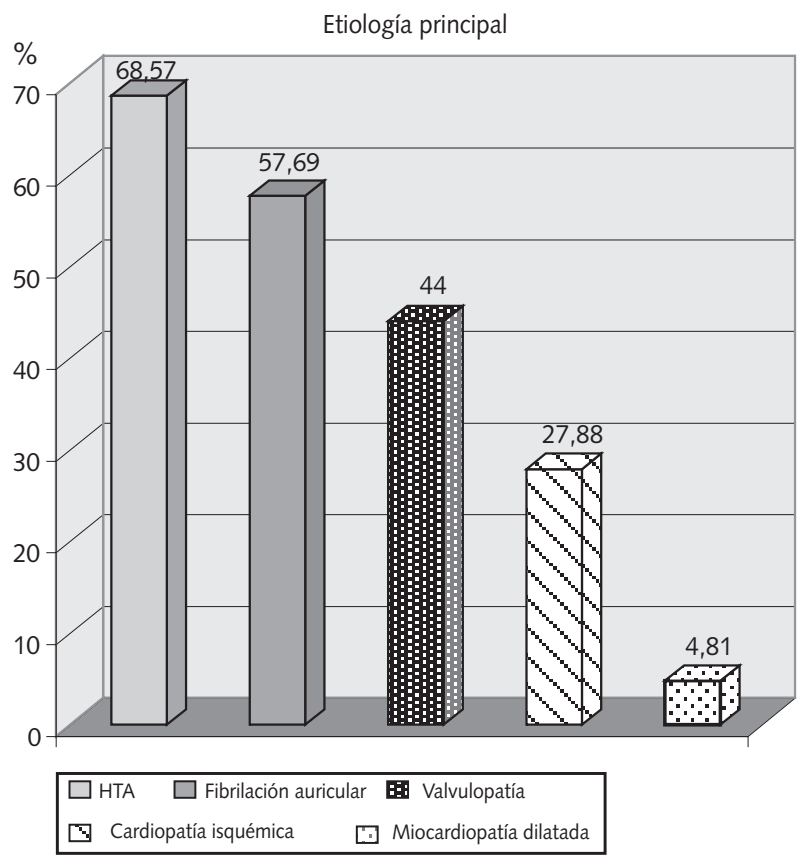

Fig. 1. Etiología principal de la insuficiencia cardiaca.

Los tipos de insuficiencia cardiaca más frecuente fueron la global $(33,65 \%)$ y la insuficiencia cardiaca izquierda $(33,65 \%)$. El $29,81 \%$ de los pacientes presentaron insuficiencia cardiaca derecha e ingresaron en edema agudo de pulmón el $2,89 \%$.

El porcentaje de los síntomas que presentaban los pacientes de la serie, basados en los criterios diagnósticos de Framingham de IC, tanto mayores como menores, se describen porcentualmente en la tabla I.

Al 55,77\% de los pacientes se les realizó un ecocardiograma para definir el tipo de disfunción ventricular. El 5,17\% de las mujeres y el 20,69\% de los hombres presentaron disfunción sistólica, existiendo diferencias significativas (p 0,023). Presentaban disfunción diastólica el $32,76 \%$ de las mujeres y el $41,38 \%$ de los hombres (sin diferencias significativas). El porcentaje de pacientes con disfunción sistólica leve fue de $33,33 \%$, con disfunción moderada el $40 \%$ y con disfunción severa el $26,66 \%$.

La infección respiratoria fue el factor etiológico que con más frecuencia $(50 \%)$ desencadenó la descompensación de la
TABLA III

TRATAMIENTO DE LA INSUFICIENCIA CARDIACA AL ALTA

\begin{tabular}{lc}
\hline \multicolumn{2}{c}{ Tratamiento al alta } \\
\hline Diuréticos & $90,38 \%$ \\
Digitálicos & $41,35 \%$ \\
IECAS y antiagregantes & $36,54 \%$ \\
ACO & $33,65 \%$ \\
Antagonistas de calcio & $27,88 \%$ \\
Antialdosterónicos & $20,19 \%$ \\
B bloqueantes & $19,23 \%$ \\
Hipolipemiantes y ARA II & $16,35 \%$ \\
Nitritos & $15,38 \%$ \\
Alfa bloqueantes & $9,61 \%$ \\
Antiarrítmicos & $4,8 \%$ \\
\hline
\end{tabular}

IC, seguida de la fibrilación auricular $(21,1 \%)$, la anemia $(6,73 \%)$, la infección urinaria $(4,81 \%)$ y la crisis hipertensiva en un $0,96 \%$.

Al alta la mayoría de los pacientes recibieron como tratamiento de fondo diuréticos (el 90,38\% de los pacientes), digitálicos en segundo lugar $(41,35 \%)$, IECAS $(36,53 \%)$, antiagregantes $(36,53 \%)$, diuréticos + IECAS $(35,58 \%)$, anticoagulación oral $(33,65 \%)$, antagonistas del calcio $(27,88 \%)$, antialdosterónicos $(20,19 \%)$, beta bloqueantes $(19,23 \%)$, hipolipemiantes y ARAII $(16,35 \%)$, nitritos $(15,38 \%)$, diuréticos + IECAS + antialdosterónicos $(12,5 \%)$, alfa bloqueantes $(9,61 \%)$ y antiarrítmicos $(4,8 \%)$, como queda reflejado en la tabla III.

La estancia media fue de 15,49 días y los reingresos alcanzaron el 26,67\% haciéndolo en el propio Servicio de Medicina Interna o en Cardiología. Los hombres reingresaron el 12,5\% y las mujeres en un $15,38 \%$ ( sin encontrar diferencias signicativas $\mathrm{p} 0,306)$.

\section{DISCUSIÓN}

La IC es una enfermedad muy prevalente en nuestro medio, con elevada morbimortalidad, que se incrementa de manera importante con la edad. Por ello es uno de los motivos de ingreso más frecuentes en los servicios de Medicina Interna, preferentemente en aquellas poblaciones más envejecidas, como la que corresponde al HCUV. Esto, junto a la frecuente comorbilidad asociada, hace que, la mayor parte de las veces, el pronóstico sea grave.

La población estudiada, en esta serie, es de edad avanzada, mayor en las mujeres, al igual que lo aportado por otros estudios realizados en servicios de Medicina Interna similares al nuestro $(10,11)$. Dado que se ha demostrado y así sucede en nuestra serie, que el sexo no influye de manera significativa en la evolución y el pronóstico de la enfermedad (12), no haremos ningún comentario al respecto.

La hipertensión arterial, es la primera causa de IC (13) y el principal factor de riesgo asociado, en nuestros pacientes, coincidiendo con otros estudios realizados en diferentes hospitales (14) y en contra de los referidos por otros (10), preferentemente en los servicios de Cardiología donde la cardiopa- 
tía isquémica es la primera causa. En nuestro estudio ésta figura en tercer lugar, después de la fibrilación auricular. Ello quizás sea debido a la edad y a las características clínicas de los pacientes que ingresan en los servicios de Medicina Interna.

La infección respiratoria es el factor desencadenante primordial de la descompensación de IC y la disnea es la causa principal de ingreso hospitalario, como en la mayoría de los estudios revisados $(10,11,14)$.

Dado que la IC suele asociarse a un deterioro de la función ventricular, preferentemente sistólica, es conveniente, para su diagnóstico y para la valoración del pronóstico y evolución, la realización de un ecocardiograma. Se practicó al 55,77\% de nuestros pacientes, porcentaje elevado con respecto a otras series revisadas (15). Este hecho llama la atención dado que, se ha demostrado, que la infrautilización de la ecocardiografía, en el manejo de los pacientes con IC, se asocia a una disminución de la supervivencia (16). En este estudio predomina la disfunción diastólica sobre la sistólica tanto en varones como en mujeres, posiblemente por el escaso porcentaje de cardiopatía isquémica y la edad tan avanzada que presenta la muestra, pero con diferencias significativas entre ambos sexos, ya que la disfunción sistólica es más frecuente en los varones, quizás debido a que la cardiopatía isquémica es más habitual en ellos.

A pesar de que tradicionalmente se ha considerado la disfunción diastólica más benigna que la disfunción sistólica, existen estudios que lo cuestionan, puesto que no han encontrado diferencias significativas en cuanto a la morbimortalidad $(17,18)$ ni a la calidad de vida al comparar pacientes con disfunción diastólica y función sistólica conservada (19).

El tratamiento de la IC varía de manera importante según el medio donde se trate al paciente y la especialidad médica que intervenga. Sería de desear un tratamiento multidisciplinario. Este se ha demostrado eficaz en la reducción de los reingresos hospitalarios, así como en el acortamiento del tiempo del ingreso y en la mejoría de la calidad de vida (20-22).

Creemos de interés destacar, tanto en nuestra serie como en otras revisadas, el gran porcentaje de pacientes sometidos a tratamiento con diuréticos y con digoxina, a pesar de que los primeros no han demostrado que favorezcan el pronóstico de la IC, aunque son eficaces (10). Por el contrario, llama la atención la infrautilización de beta bloqueantes en los pacientes tratados en los servicios de Medicina Interna, si lo comparamos con los tratados en Cardiología (11,23-25).

En la serie de Permanyer (26) sólo el 4\% de los pacientes con IC eran tratados con betabloqueantes y el porcentaje dado por el Grupo de Trabajo de Insuficiencia Cardiaca de la Sociedad Española de Medicina Interna (SEMI) (27), era del 10\%, frente al $18,23 \%$ de nuestra serie.

Las causas pueden ser numerosas. Probablemente sea debido al tipo de pacientes que ingresan en los diferentes servicios, junto a la dificultad de asegurar un diagnóstico correcto, el temor a producir descompensaciones severas en la comorbilidad, preferentemente de tipo respiratorio y diabetes mellitus, o al temor de desencadenar efectos secundarios propios de esta medicación (28). Estos pacientes, en nuestra serie, representaban al $28 \%$ de la muestra, valor que no justifica la escasa utilización de esta medicación, dado que, además, los beta bloqueantes cardioselectivos apenas modifican la resistencia de las vías aéreas, ni provocan alteraciones respira- torias clínicamente manifiestas, en pacientes con enfermedad pulmonar obstructiva crónica (29).

Está plenamente demostrado el efecto beneficioso, tanto sobre la reducción de mortalidad como del número de reingresos y sobre la calidad de vida, de los betabloqueantes, así como con de los IECAs, que junto a los antialdosterónicos, son parte fundamental del tratamiento $(30,31)$.

Esto queda reflejado en numerosos estudios. El estudio CIBIS II (32), mediante el empleo de bisoprolol, demuestra, además de la reducción de la mortalidad, disminución del riesgo de reingreso hospitalario, que en nuestro estudio fue del $26,67 \%$, obsteniéndose aún mejores resultados en el estudio COPERNICUS (33) que incluye pacientes con IC clase IV de NYHA y con fracción de eyección menor del $25 \%$.

El estudio MERIT HF estudió 3991 pacientes con insuficiencia cardiaca congestiva en clase funcional II-III de la NYHA y fracción de eyección menor del 40\%. Se redujo la mortalidad en un 34\% y disminuyó el riesgo de muerte súbita y de mortalidad por empeoramiento de la insuficiencia cardiaca (34).

Recientemente el estudio COMET (35) ha analizado qué tipo de betabloqueantes ofrecen más beneficio al paciente, observándose que con el bloqueo completo $(\mathrm{B} 1, \mathrm{~B} 2)$, frente al parcial B1 selectivo, se mejora la supervivencia significativamente en un $17 \%$ y se reduce la mortalidad cardiovascular en un $21 \%$.

Así mismo el estudio SENIORS demuestra la eficacia de un beta bloqueante (nebivolol) en pacientes ancianos con insuficiencia cardiaca crónica con función sistólica conservada, que se acerca mucho a los pacientes de nuestra muestra $(36,37)$.

El coste-efectividad y el coste-beneficio del tratamiento de la IC con beta bloqueantes, según Diez Manglano (38), es la terapéutica con carvedilol la más eficaz y bisoprolol la más coste-efectiva y con mayor coste-beneficio.

Para actuar sobre el remodelado de la pared ventricular en pacientes con IC leve (CARMEN) $(39,40)$ se ha ensayado la asociación de betabloqueantes con IECAs, observándose una inversión significativa del remodelado del VI en estos pacientes con respecto a los tratados solo con IECAs y sin encontrar más efectos adversos, planteando el estudio CIBIS III (41) qué es mejor, si empezar el tratamiento con IECA y luego añadir el beta bloqueante o al revés.

Comentar que los diferentes IECAS han demostrado efectos beneficiosos, como ya se ha dicho, aunque con distinto nivel de evidencias según el fármaco (42).

En cuanto al tratamiento con antialdosterónicos también hay estudios que demuestran su beneficio reduciendo la mortalidad global y la frecuencia de hospitalización y muerte de causa cardiovascular $(43,44)$. Por ello deben formar parte importante en la terapeútica, junto con los IECAs y betabloqueantes. Recientemente se ha demostrado su utilidad en fases tempranas de pacientes con enfermedad coronaria aguda.

El porcentaje de pacientes con anticoagulación oral, $33,65 \%$, pensamos que es bajo, pero así mismo está en concordancia con lo observado en otros estudios, como el realizado por Román-Sánchez y cols. (11) en los servicios de Medicina Interna de 51 hospitales de España, que es del 32\%, a pesar de existir estudios que demuestran su utilidad para la prevención de la enfermedad tromboembólica, en aquellos pacientes con IC que requieren ingreso y no están previamen- 
te anticoagulados (38). El 31,73\% de los pacientes de nuestra serie que presentaban fibrilación auricular, estaban anticoagulados, el $19,23 \%$, antiagregados y un $6,73 \%$, tratados con antiagregantes y anticoagulantes.

Casi un tercio de los pacientes con insuficiencia cardiaca reingresaron por descompensacion de su enfermedad en diversos servicios hospitalarios (Medicina Interna o Cardiología), proporción demasiado elevada, quizás en relación con el tipo de tratamiento que reciben los pacientes al alta (14). El Grupo de Trabajo de Insuficiencia Cardiaca de la SEMI estima que el $63 \%$ de los pacientes del estudio había tenido un ingreso previo y que una vez diagnosticados el $30 \%$ reingresaba en los primeros 6 meses.

Por ello, y como anteriormente expusimos, creemos que con un tratamiento multidisciplinario podrá conseguirse una reducción de los reingresos hospitalarios, así como en el acortamiento del tiempo del ingreso y una mejoría de la calidad de vida (39-41).

Como conclusión diremos que el diagnóstico y el tratamiento de la IC en el servicio de Medicina Interna es aceptable, pero es importante recalcar el interés que tiene el realizar un mejor diagnóstico mediante estudio ecocardiográfico para catalogar y valorar el tipo de IC, así como potenciar el uso de betabloqueantes, IECAs e inhibidores de la aldosterona. La experiencia en su manejo hará que poco a poco desaparezcan los recelos hacia sus complicaciones y efectos secundarios. Esto redundará en la supervivencia y mejora de la calidad de vida de los pacientes, evitando, además, reingresos, por lo que supone de riesgo para ellos, dada la edad y la comorbilidad tan elevada que presentan.

\section{Bibliografía}

1. Ho KK, Pinsky JL, Kannel WB, Levy D. The epidemiology of Heart failure; the Framinghan study. J Am Coll Cardiol 1993; 22: 6A-13.

2. Rodríguez-Artalejo F, Banegas Banegas JR, Guallar-Castillón P. Epidemiología de la insuficiencia cardiaca. Rev Esp Cardiol 2004; 57: 163 170.

3. Cortina A, Reguero J, Segovia D, Rodríguez Lambert JL, Cortina R, Arias JC, et al. Prevalence of Heart failure in Asturias. Am J Cardiol 2001; 87: 1417-9.

4. Cleland JGF, Khand A, Clark A. The Heart failure epidemic: exactly how big is it? Eur Heart J 2001; 22: 623-6.

5. Stewart S, Maclntyre K, Hole DJ, Capewell S, McMurray JJ. More "malignant" than cancer? Five-year survival following a first admission for heart failure. Eur J Heart Fail 2001; 3: 315-22.

6. Poses RM, McClish DK, Smith WR, et al. Results of report cards for patients with congestive heart failure depend on the meted used to adjust for severity. Ann Intern Med 2000; 133: 10-20.

7. McKee PA, Castelli WP, McNamara PM, Kannel WB. The natural history of congestive heart failure: the Framingham study. N Engl J Med 1971; 285: 1441-6.

8. Caruana L, Petrie MC, Davie AP, McMurray JJV. Do patients with suspected heart failure and preserved left ventricular systolic function suffer from diastolic heart failure or from misdiagnosis? A prospective descriptive study. BMJ 2000; 101: 2118-21.

9. Ho KK, Anderson KM, Kannel WB, Grossman W, Levy D. Survival after the onset of congestive heart failure in Framingham Heart Study subjects. Circulation 1993; 88: 107-15.

10. Torres-Gárate R, Álvarez Rodríguez E, Segoviano Mateo R, Gutierrez Larrainzar A, Lozano Tonkin C, Calvo Manuel E. Clinical and therapeutic characteristics in patients with heart failure cared in a tertiary hospital. Rev Clin Esp 2005; 205: 305-6.

11. Román Sánchez P, Conthe P, García-Alegría J, Forteza- Rey J, Montero M, Montoto C. Factors influencing medical treatment of Heart failure patients in Spanish internal medicine departments: a nacional survey. QJM 2005; 98: 127-38.

12. Varela Román A, Grigorian Shamagian L, Bandín Diéguez MA, Rigueiro Veloso P, González Juanatey JR. Influencia del sexo en la mortalidad a largo plazo de pacientes hospitalizados por insuficiencia cardiaca con función sistólica conservada o deprimida. Rev Esp Cardiol 2005; 58 : 1171-80.

13. Havranek EP, Wolfe P, Masoudi FA, Rathore SS, Krumholz HM, Ordin DL. Provider and hospital characcteristics associated with geografphic variation in the evaluation and management of elderly patients with heart failure. Arch Intern Med 2004; 164: 1186-91.

14. Recio Iglesias J, Alegre Martin J, Fernandez de Sevilla T. A prospective study on patients with Heart failure admitted to the internal medicine department. Rev Clin Esp 2004; 204: 362-4.
15. Álvarez Frías MT, Gutiérrez Dubois J, Alonso Martínez JL, Solano Ramírez M, González Arencibia C, Etxegaray Agara M. Quality management in patients with Heart failure incidence an Internal Medicine Department. An Med Interna (Madrid) 2005; 22: 307-8.

16. Senni M, Rodeheffer RJ, Tribouilloy CM, et al. Use of echocardiografphy in the management of congestive heart failure in the community. J Am Coll Cardiol 1999; 33: 164-70.

17. Smith GL, Masoudi FA, Vaccarino V, Radford MJ, Krumholz HM. Outcomes in heart failure patients with preserved ejection fraction: mortality, readmisión, and funcional decline. J Am Coll Cardiol 2003; 41(9): 1510-8.

18. Badano LP, Albanese MC, De Biaggio P, Rozbowsky P, Miani D, Freco $\mathrm{C}$, et al. Prevalence, clinical characteristics, quality of life, and prognosis of patients with congestive heart failure and isolated left ventricular diastolic dysfunction. J Am Soc Echocardiogr 2004; 17: 253-261.

19. Jaarsma T, Halfens R, Abu-Saad HH, Dracup K, Stappers J, van Ree J. Quality of life in older patients with systolic and diastolic heart failure. Eur J Heart Fail 1999; 1: 151-160.

20. Ducharme A, Doyon O, White M, Rouleau JL, Brophy JM. Impact of care at a multidisciplinary congestive heart failure clinic: a randomized trial. CMAJ 2005; 173: 53-4.

21. Piepoli MF, Villani GQ, Aschieri D, Bennati S, Groppi F, Pisati MF, et al. Multidisciplinary and multisetting team management programme in heart failure patients affects hospitalisation and costing. Int J Cardiol 2005 (en prensa).

22. López-Sendón J. ¿Trabajo en equipo, unidades de insuficiencia cardíaca o especialidad de insuficiencia cardíaca? Rev Esp Cardiol 2004; 57: 1136-1138.

23. Stawicki S, Roik M, Jasik M, Kosior D, Scislo P, Kochanowski J, et al. Differences between cardiologist and internist in the management of Heart failure. Medical guidelines compared with every-day practice. Kardiol Pol 2004; 61: 431-9.

24. Jaarsma T, Haaijer-Ruskamp FM, Sturm H, Van Veldhuisen DL. Management of Herat failiru in The Netherlands. Eur J Herat Fail 2005; 16: 371-5.

25. Patel JA, Fotis MA. Comparison of treatment of patients with congestive Herat failure by cardiologist versus noncardiologists. Am J Health Syst Pharm 2005; 62: 168-72.

26. Permanyer G, Soriano N, Brotons C, Moral I, Pinar J, Cascant P, et al. Características basales y determinantes de la evolución en pacientes ingresados por insuficiencia cardiaca en un hospital general. Rev Esp Cardiol 2002; 55: 571-578.

27. Grupo de Trabajo de Insuficiencia Cardiaca de la Sociedad Española de Medicina Interna (SEMI). Insuficiencia cardiaca en los servicios de medicina interna (estudio SEMI-IC). Med Clin (Barc) 2002; 118: 605610. 
28. Manzano I, Sánchez Mateo D, Rodríguez González P. La insuficiencia cardiaca en la práctica asistencial del internista. Rev Clin Esp 2005; 205: 305-6.

29. Salpeter SR, Ormiston T, Salpeter E, Poole P, Cates C. Cardioselective beta-blockers for chronic obstructive pulmonary disease. Cochrane Database Sist Rev 2002; 2: CD003566.

30. Remme WJ, Swedberg K. Guidelines for the diagnosis and treatment of chronic heart failure. Task Force for the diagnosis and treatment of chronic heart failure. European Society of Cardiology. European Society of Cardiology. Eur Heart J 2001; 22: 1527-1560.

31. Shekelle PG, Rich MW, Morton SC et al. Efficacy of angiotensina-converting enzyme inhibitor and beta-blockers in the management of left ventricular systolic dysfunction according to race, gender, and status. A meta-analysis of major clinical trials. J Am Coll Cardiol 2003; 41: 152938.

32. CIBIS II Investigators. The Cardiac Insufficiency Bisoprolol Study II: a randomised trial. Lancet 1999; 353: 9-13.

33. Packer M, Coats AJ, Fowler MB, Katus HA, Krum H, Mohacsi P, et al, for the Carvedilol Prospective Randomized Cumulative Survival Study Group. Effect of carvedilol on survival in severe chronic heart failure. $\mathrm{N}$ Engl J Med 2001; 344: 1651-1658.

34. Hjalmarson A, Goldstein S, Fagerberg B, Wedel H, Waagstein F, Kjekshus J, et al, for teh MERIT HS Study Group. Effects of controlled-release metoprolol on total mortality, hospitalizations, and web-being in patients with heart failure: the Metoprolol CR/XL Randomized Intervention Trial in congestive heart failure (MERIT HF). JAMA 2000; 283: 1295-1302.

35. Poole-Wilson PA, Cleland JG, Di Lenarda A, Hanrath P, Komajda M, Metra M, et al. Rationale and design of the carvedilol or metoprolol European trial in patients with chronic heart failure: COMET. Eur J Heart Fail 2002; 4: 321-329.

36. Shibata MC, Flather MD, Bohm M, Borbola J, Cohen-Solal A, Dumitrascu D, et al. Study of the effects of nebivolol intervention on outcomes and rehospitalisation in seniors with heart failure. Rationale and design. Int J Cardiol 2002; 86: 77-85.

37. Study of the Effects of Nebivolol Intervention on Outcomes and Rehospitalisation in Seniors with heart failure. SENIORS. Eur Heart J 2005;

\section{6: $215-225$}

38. Diez Manglano J. Análisis coste-efectividad y coste-beneficio del tratamiento con bloqueadores beta de la insuficiencia cardíaca congestiva en España. Rev Clin Esp 2005; 205: 140-56.

39. Remme WJ, the CARMEN Steering Committee and Investigators. The Carvedilol and ACE-inhibitor Remodelling Mild Heart Failure EvaluatioN trial. Rationale and design. Cardiovasc Drugs Ther 2001; 15: 6077.

40. Remme WJ, Swedberg K. Task force for the diagnosis and treatment of chronic Heart failure of the European Society of Cardiology. Comprehensive guidelines for the diagnosis and treatment of chronic heart failure. Eur J Heart Fail. 2002; 4: 11-22.

41. CIBIS III. Eur J Heart Fail 2004; 6: 493-500.

42. Baños Alonso V, Escobar Cervantes C, Calderón Montero A. ARA II en insuficiencia cardiaca congestiva. Coincidencias y divergencias. ¿Efecto de clase? Rev Clin Esp 2005; 205: 499-506.

43. Zannad F, Alla F, Dousset B, Pérez A, Pitt B, RALES Investigators. Limitation of excessive extracellular matriz turnover may contribute to survival benefit of spironolactone therapy in patients with congestive heart failure: insights from the randomized Aldactone Evaluation Study (RALES). Circulation 2000; 102: 2700-2706.

44. Pitt B, Remme W, Zannad F, Neaton J, Martinez F, Roniker B, for the Eplerenone Post-Acute Myocardial Infarction Heart Failure Efficacy and Survival Study (EPHESUS) Investigators. Eplerenone, a Selective Aldosterone Blocker, in patients with left ventricular dysfunction after myocardial infarction. N Engl J Med 2003; 348: 1309-21.

45. Samama MM, Cohen AT, Dammon JY et al. A comparison of enoxaparin with placebo for the prevention of venous thromboembolism in acutely ill medical patients. Prophylaxis in MEDICAL patients with enoxaparin Study group. N Engl J Med 1999; 341: 793-800.

46. Ducharme A, Doyon O, White M, Rouleau JL, Brophy JM. Impact of care at a multidisciplinary congestive heart failure clinic: a randomised trial. CMAJ 2005; 173: 53-4.

47. Piepoli MF, Villani GQ, Aschieri D, Bennati S, Groppi F, Pisati MF, Rosi A, Capucci A. Multidisciplinary and multisetting team management programme in heart failure patients affects hospitalisation and costing. Int J Cardiol 2005 (en prensa). 